Proc. of the 15th Int. Workshop on Slow Positron Beam Techniques and Applications, Prague, September 2-6, 2019

\title{
Effect of Detector Material and Size on Pile-Up Elimination in Positron Burst Measurement
}

\author{
Fuyan LiU ${ }^{a, b}$, Yinguie WAnG ${ }^{a}$, Xingzhong $\mathrm{CAO}^{a, b}$ And BaOyi Wang ${ }^{a, b, *}$ \\ ${ }^{a}$ Institution of High Energy Physics, Chinese Academy of Sciences, Yuquan Road 19B, 100049 Beijing, China \\ ${ }^{b}$ University of Chinese Academy of Sciences, Yuquan Road 19A, 100049 Beijing, China
}

\begin{abstract}
We have developed a space distribution method for intense sub-nanosecond or even picosecond positron burst detection using a detector array. In this method, piled signals will be generated when more than one photon of the burst is captured by the same detector cell. The way of setting energy threshold of $511 \mathrm{keV}$ could eliminate most of piled signals, but the counts of piled signals that have lower energy than the threshold are not affected. In this paper, a Geant4 model is built to investigate the dependence of these low-energy piled signals counts on scintillator materials, as well as the size of the detector. The results can provide theoretical support for designing suitable array detectors in practice.
\end{abstract}

DOI: 10.12693/APhysPolA.137.152

PACS/topics: 78.70.Bj, 07.77.Ka

\section{Introduction}

Usually, positron annihilation lifetime spectroscopy (PALS) is performed by collecting positron annihilation events one by one, and the time taken to obtain a spectrum can be anywhere from tens of minutes to days, depending on the source intensity and the apparatus structure. Based on the positron trapping technique, a single-shot positron annihilation lifetime spectroscopy (SSPALS) method has been developed for completing the positron lifetime spectrum using a single positron shot [1-4]. In order to avoid saturation of the detector, a fast Cherenkov radiator, coupled to a fast response photomultiplier, is used to detect the pulse of positrons in this approach [1-4]. Recently, higher intensity positron bursts have been produced with the developments of femtosecond laser induced positron technique [5], and huge number of gamma photons are released in several picoseconds after positrons annihilation. Using such highbrightness positron bursts, it is possible to characterize the evolution of electronic structure and defect configurations in materials, at nanosecond time scale [6, 7]. In order to detect these bursts, a method of space distribution detection was proposed in Ref. [8], and a scintillator detector array, composed of enough independent detector cells, is designed for measurement. In this method, all detector cells are arranged in a sufficiently large spherical surface, and each photon in the burst can be recognized by the spatial solid angle of the detector cell.

In fact, because the launched direction of the photons is random, it is probable that a detector unit captures one more gamma ray and then generates a piled burst signal during one burst. Preliminary results have shown that the time duration of the gamma burst are underesti-

*corresponding author; e-mail: wangboy@ihep.ac.cn mated, due to the pile-up effect, and the more serious the pile-up is, the greater the deviation is. We find the total deposited energy is more than $511 \mathrm{keV}$ for most piled signals, and these signals could be eliminated by setting an energy threshold of $511 \mathrm{keV}$. But because the energy depleted in the detector by the photons is random, there are still some piled signals whose total deposited energy is lower than $511 \mathrm{keV}$, so that setting threshold has no effect on these signals. It is known that differences in scintillation detector material and size will affect the deposited energy distribution of photons [9-11], and thus influence the counts of piled signal in energy zone of $0-511 \mathrm{keV}$. Therefore, in present work, the detection probability of piled burst signals in $0-511 \mathrm{keV}$ energy region is studied while setting different types of scintillators and sizes for the detector.

\section{Monte Carlo simulation method}

In this study, the Geant4 Monte Carlo toolkit is applied to simulate the course of detecting the gamma burst. Based on $\mathrm{C}++$ language, Geant4 is developed for simulating the interaction of particles through matter [12]. Since the relative independence of each detector unit in the array, a single detector is used for the burst measurement in our simulations. Instead of measuring positron burst lifetimes, the main purpose of the simulation is to study the effect of the detector configuration on the piled signals counts after setting $511 \mathrm{keV}$ threshold. Therefore, only the gamma-ray interactions with the detector are considered in simulations, neglecting the annihilation process of positrons. In our model then the burst, composed of $511 \mathrm{keV}$ gamma photons, is set as the source with an intensity of $2000 \mathrm{\gamma} /$ burst, corresponding to the actual burst intensity. In addition, the Livermore low-energy physics model is used in simulations to track the photons as well as other secondary particles from interactions, and 150,000 bursts are emitted for 
each simulation to ensure enough statistics. During detection every burst, we record the number of photons detected by the detector and the total deposited energy in the detector.

When a $4 \mathrm{~mm} \times 4 \mathrm{~mm} \times 10 \mathrm{~mm}$ (thick) LYSO crystal is set as the detector, with the $4 \times 4 \mathrm{~mm}^{2}$ side facing the source, Fig. 1 shows the counts distributions of different burst signals detected. It can be seen that the detector could detect different types of burst signals at the same place, including $1 \gamma, 2 \gamma, 3 \gamma$, and above $3 \gamma$ signals which respectively means that the detector simultaneously captures one photon, two photons, three photons, and more than three photons in the burst. In Fig. 1, as the detector distance increases, the counts of $1 \gamma, 2 \gamma$, and $3 \gamma$ signals rise to the maximum and then fall gradually, with the maximum values corresponding to different positions but the number of above $3 \gamma$ signals are decreasing to zero. Additionally we can also find that the relative proportions of different burst signals change with the distance, indicating the different pile-up level at different locations.

By setting an energy threshold of $511 \mathrm{keV}$, Fig. 2 shows that most of piled burst signals could be "deleted". It can be also seen in Fig. 2 that because the total energy deposited by all photons detected in the burst may be lower than threshold, there are still a small number of piled signals which could not be excluded. Only when the gamma ray depletes more energy in the detector, the total deposited energy of the piled signal can possibly exceed $511 \mathrm{keV}$, and more piled signals can be eliminated by setting threshold. Moreover, the energy distribution of gamma rays detected is related to the materials and sizes of scintillation detector. Therefore, in our Geant 4 model, different scintillators and multiple size are set for the detector to detect bursts, and the counts of piled burst signals in the energy region of $0-511 \mathrm{keV}$ are recorded accordingly.

\section{Results and discussion}

In every simulation, the distance from the detector to the source varies as the detector changes and is always set to the position where the probability of $1 \gamma$ burst signals is maximum (as seen in Fig. 1), in order to ensure the same pile-up level for different detectors. In other words, the high-efficiency detector should be placed further away to detect as many piled signals as the low-efficiency one. In addition, it is noted that the simulated values of the probability are calculated as the ratio of particular signal counts to the total burst events generated.

For scintillator materials trials, the detector size is fixed to $4 \times 4 \times 10 \mathrm{~mm}^{3}$ with the $4 \times 4 \mathrm{~mm}^{2}$ cross profile facing the source, and a series of commonly used crystal (including NaI, BaF2, GSO, LYSO, LSO, and PWO) with different densities are set as the scintillator of the detector. Figure 3a shows the detection probabilities of different piled burst signals whose deposited energy is less than $511 \mathrm{keV}$. It can be seen that the probabilities of $2 \gamma, 3 \gamma$, and above $3 \gamma$ piled signals decrease as the

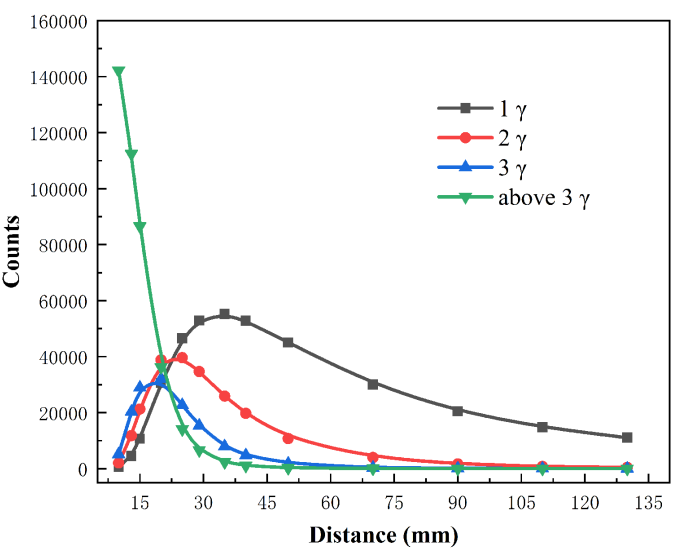

Fig. 1. Counts distribution of burst signals as a function of distance from detector to the source.

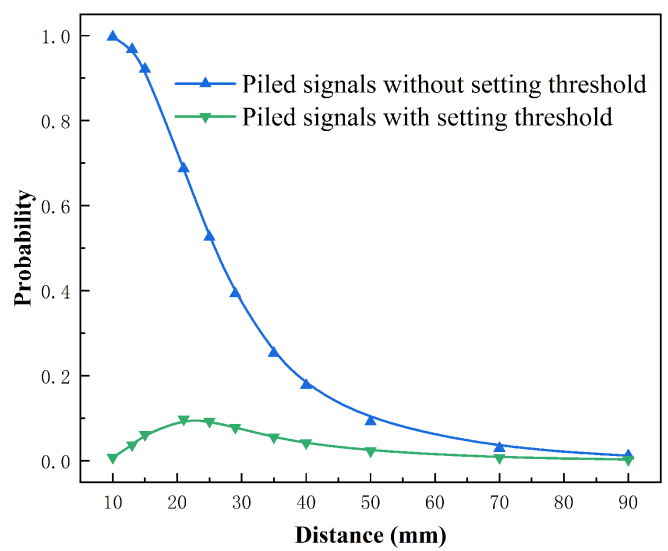

Fig. 2. Detection probabilities of piled signals with and without setting threshold.

scintillator density increases, and the $2 \gamma$ piled burst signals is dominant in the piled signals of $0-511 \mathrm{keV}$. Additionally, we find the probability of all piled signals could be reduced by $\approx 10 \%$ in the energy range of $0-511 \mathrm{keV}$ and this reduction will be larger for higher density of the detector crystal.

The reduction of piled signals could be interpreted by the different absorption coefficient for gamma photons in different types of scintillators. Table I shows the physical properties of these commonly available scintillator materials, listed in order of increasing density. In general, high density scintillator has a high atomic number which results in big absorption coefficient for gamma rays, and then the photons will deplete more energy in the detector. It could also be demonstrated in Fig. $3 \mathrm{~b}$ that the ratio of the Compton counts to all counts detected falls as the density of the detector material increases, when detecting the single $511 \mathrm{keV}$ gamma rays.

Additionally, we also calculate the ratio of the piled signals and the single gamma $(1 \gamma)$ signals after setting $511 \mathrm{keV}$ threshold, which is necessary to evaluate the effect of the method on removing piled events from the final 

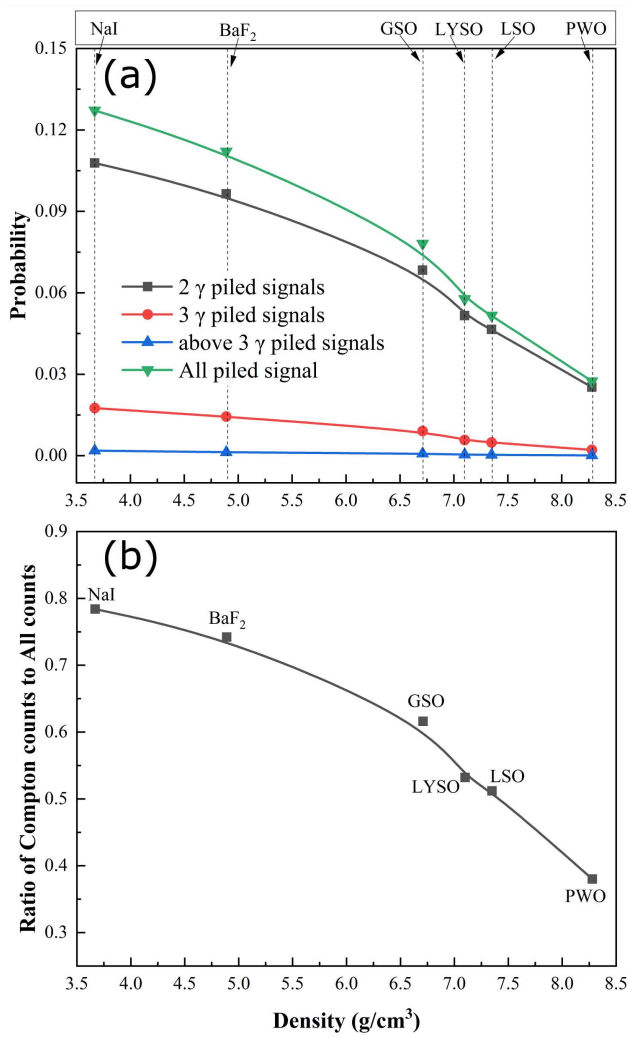

Fig. 3. With changing the scintillator materials, (a) the detection probabilities of different piled burst signals in $0-511 \mathrm{keV}$ energy region, (b) and the ratio of the Compton counts to all counts detected for single $511 \mathrm{keV}$ photon.

TABLE I

Physical properties of some common scintillator crystals.

\begin{tabular}{c|c|c|c|c|c|c}
\hline \hline Crystal & $\mathrm{NaI}$ & $\mathrm{BaF}_{2}$ & $\mathrm{GSO}$ & $\mathrm{LYSO}$ & $\mathrm{LSO}$ & $\mathrm{PWO}$ \\
\hline $\begin{array}{c}\text { density }\left[\mathrm{g} / \mathrm{cm}^{3}\right] \\
\text { eff. at. number }\end{array}$ & 3.69 & 4.89 & 6.71 & 7.10 & 7.35 & 8.28 \\
& 53 & 59 & 60 & 65 & 76
\end{tabular}

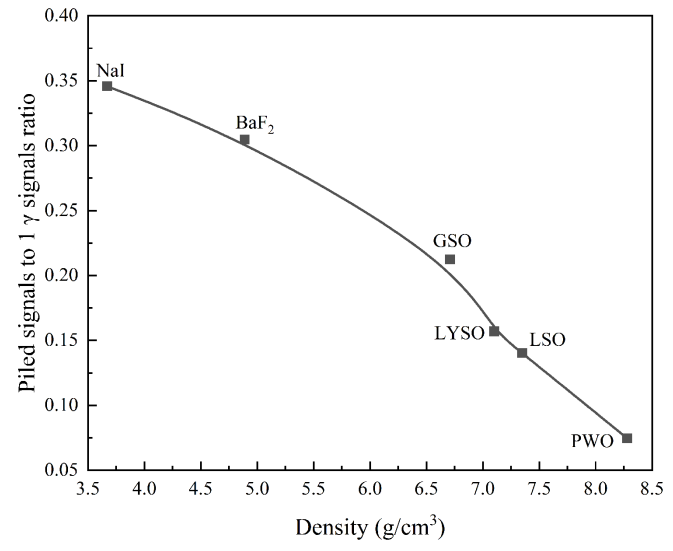

Fig. 4. The ratio of piled signals to $1 \gamma$ signals after setting $511 \mathrm{keV}$ energy threshold as a function of the scintillator materials density.
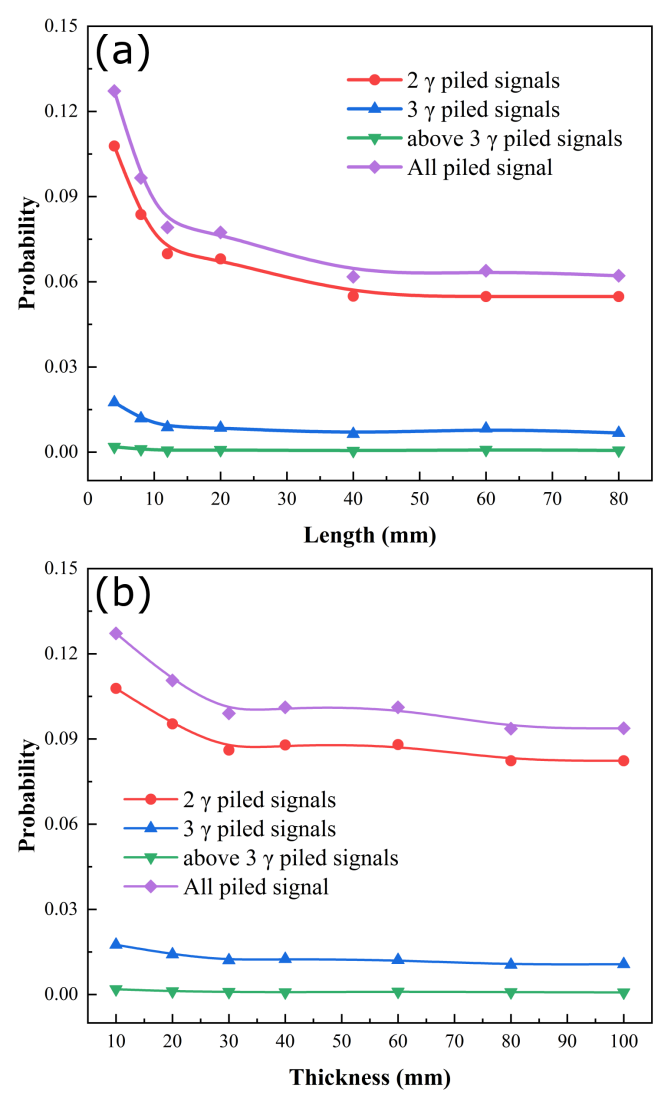

Fig. 5. The detection probabilities of different piled burst signals in 0-511 keV energy region, (a) by changing the cross profile length of the detector with $10 \mathrm{~mm}$ thickness, (b) and changing the detector thickness with a fixed cross profile of $4 \times 4 \mathrm{~mm}^{2}$.

positron lifetime spectrum. As shown by the results in Fig. 4 , the piled signals to $1 \gamma$ signals ratio drops quickly with increasing the density of the crystal material, indicating positron burst lifetimes could be measured more correctly using high-density scintillators neglecting the time performance.

Besides the scintillator material, the size of the detector is another factor we considered in simulation, and $\mathrm{NaI}$ is used as the detector crystal. As seen the results in Fig. 5, enlarging the detector size could also lessen the detection probabilities of piled burst signals whose deposited energy is less than $511 \mathrm{keV}$. It is because with a big-sized detector, the chances of photons interacting with the detector material increase, and more energy will be lost in the detector. Furthermore, the probability of all piled signals in the energy range of $0-511 \mathrm{keV}$ could be reduced by $6.5 \%$ by changing the cross-section length in Fig. 5a, compared to that of the original $4 \times 4 \times 10 \mathrm{~mm}^{3}$ size. Although, by changing the detector thickness, the reduction of all piled signals probability in Fig. $5 \mathrm{~b}$ is only $3.3 \%$, indicating that the detector length is more effective than thickness on the energy distribution of the piled burst signals. 


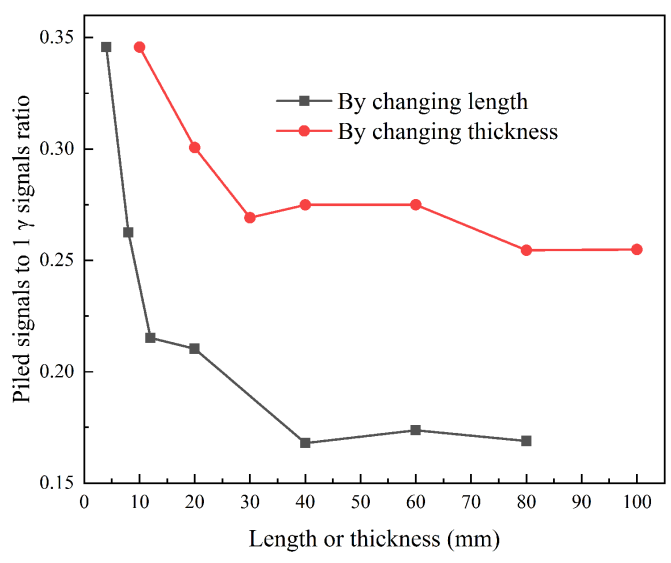

Fig. 6. The ratio of piled signals to $1 \gamma$ signals after setting $511 \mathrm{keV}$ energy threshold, by changing the length (black line) and changing the thickness (red line) of the detector. The other settings are the same as those in Fig. 5.

Figure 6 shows that the ratio of piled signals to $1 \gamma$ signals varies as a function of the size of the detector. With enlarging the detector size, the ratio is decreasing. Further after setting $511 \mathrm{keV}$ threshold, the ratio is smaller in the case of changing the detector length compared to changing the thickness, and this is beneficial for the accurate measurement of positron lifetimes in the burst.

It can be concluded from the overall analysis that the counts of piled signals in the energy range of $0-511 \mathrm{keV}$ depend on the detector material and size, and the most influential factor is the scintillator material. Compared with the initial $4 \times 4 \times 10 \mathrm{~mm}^{3} \mathrm{NaI}$ detector, the counts of piled signals that have energy lower than $511 \mathrm{keV}$ could be reduced by at least $10 \%$ for different types of scintillator. The reduction of these piled signals is at most $6.5 \%$ and at most $3.3 \%$ by changing the cross profile length and the thickness of the detector, respectively. Moreover, it should be pointed out that light yield, time decay constant, and other time properties of scintillators are not considered in simulation, and are assumed to be acceptable when focusing on the energy properties. In fact, owing to the improvement of the detector efficiency when the detector is set as high-density materials or have a big size, more piled signals could be eliminated after setting threshold. Further, the energy resolution is under another consideration when we actually design the piled signals removal circuit, so our next work is to determine detector parameters by balancing the resolution and efficiency performances of the detector.

\section{Conclusion}

In this study, we simulate the effect of the scintillator detector material and size on the detection probabilities of piled burst signal with energy less than $511 \mathrm{keV}$ in gamma burst detection using Geant4 code. The results show the counts of piled signals in 0-511 keV energy region could be reduced by using high density scintillator material with a high atomic number or enlarging the detector size, and the crystal material has the greatest effect, reducing the counts by at least 10\%. From the results, we can find that when the detectors in the array used high density and atomic number scintillators with a big size, there will be more piled burst signals whose deposited energy exceeds $511 \mathrm{keV}$, and these piled signals could be eliminated by setting energy threshold of $511 \mathrm{keV}$. Furthermore, the calculated results will provide a quantitative theoretical basis for potentially developing a better detector array system to eliminate pile-up in positron burst measurement.

\section{Acknowledgments}

This work was supported in part by the Scientific Instrument Developing Project of the Chinese Academy of Science (YJKYYQ20180007).

\section{References}

[1] D.B. Cassidy, S.H.M. Deng, H.K.M. Tanaka, A.P. Mills, Appl. Phys. Lett. 88, 194105 (2006).

[2] D.B. Cassidy, A.P. Mills, Nucl. Instrum. Methods Phys. Res. A 580, 1338 (2007).

[3] A.M. Alonso, B.S. Cooper, A. Deller, D.B. Cassidy, Nucl. Instrum. Methods Phys. Res. A 828, 163 (2016).

[4] A. Deller, Nucl. Instrum. Methods Phys. Res. A 922, 91 (2019).

[5] T. Xu, B. Shen, J. Xu, S. Li, Y. Yu, J. Li, X. Lu, C. Wang, X. Wang, X. Liang, Y. Leng, R. Li, Z. Xu, Phys. Plasmas 23, 33109 (2016).

[6] C. Hugenschmidt, J. Phys. Conf. Series 791, 012002 (2017).

[7] J. Mayer, C. Hugenschmidt, K. Schreckenbach, Phys. Rev. Lett. 105, 207401 (2010).

[8] B.Y. Wang, P. Kuang, F.Y. Liu, Z.J. Han, X.Z. Cao, P. Zhang, Nucl. Instrum. Methods Phys. Res. A 885, 119 (2018).

[9] F.A. Ugur, EPJ Web Conf. 100, 03006 (2015).

[10] G.R. Choppin, J. Liljenzin, J. Rydberg, Radiochemistry and Nuclear Chemistry, ButterworthHeinemann, Woburn 2002.

[11] V.M. Gerrish, Semiconductors and Semimetals, Elsevier, 1995.

[12] S. Agostinelli, J. Allison, K. Amako, et al., Nucl. Instrum. Methods Phys. Res. A 506, 250 (2003). 